\title{
Determination of Anxiety Levels in Pregnant Women During Covid-19 Pandemic
}

\author{
Basak Cingillioglu ${ }^{1}$, Erhan Akturk ${ }^{1}$, Ferhat Yasin Ozkaya ${ }^{1}$, Burak Arslan ${ }^{1}$, Simten Genc ${ }^{1}$, \\ Huseyin Turhan Ozkan ${ }^{1}$, Aydin Kilinc ${ }^{1}$, Bora Taspinar ${ }^{1}$, Elif Dilasa Pala Kose ${ }^{1}$, Veli \\ Mihmanli $^{1}$, and Ahmet Kilickaya ${ }^{1}$ \\ ${ }^{1}$ Okmeydani Training and Research Hospital
}

July 7,2020

\begin{abstract}
Objective: To examine the anxiety caused by COVID-19 pandemic and antenatal care quality of pregnant women during the isolation times. Study Design: Single center cross-sectional study. Setting: Okmeydani Training and Research Hospital. Istanbul, Turkey Population: We studied 174 pregnant, and 117 non-pregnant patients between 05 May (after the start of restrictions) and 31 May (the date restrictions eased) during the covid 19 outbreak. Methods: The data was obtained from both qualitative and quantitative methods using the State-trait anxiety inventory (STAI) and life style change questionnaire. Main outcomes and measures: We assesed anxiety levels, behavioral changes and antenatal care quality of pregnant women during Covid-19 pandemic. Results: Pregnant women had higher anxiety STAI state (STAI-S) scores than the nonpregnant women $(43,67 \pm 10,77$ vs. $39,71 \pm 9,55, \mathrm{p}=0,01)$. The STAI trait (STAI-T) anxiety scores were similar between the two groups $(43,57 \pm 8,07 \mathrm{vs} 43,07 \pm 9,41, \mathrm{p}=0,74)$. In pregnant women under age 20, STAI-S score was lower $(35,86 \pm 2,13, \mathrm{p}=0,031) .60$ of pregnant women were in first trimester, 53 were in second and 61 were in third trimester. STAI-S and STAI-T scores were not statistically different between trimester groups. Cancelling appointments of prenatal care and the fear of going to hospital is obviously high in third trimester $(46,9 \%, \mathrm{p}=<0,001 ; 41,5 \%, \mathrm{p}=0,002)$. Conclusion: In pregnant women, fear and anxiety felt even more due to quarantine period for covid 19. Evaluation and disseminated use of online health services may be helpfull in patient information and empowerment for situations such as pandemic and isolation times.
\end{abstract}

\section{Introduction:}

Covid-19 which was initially perceived as a regional "epidemic" affecting China and its surroundings, began to cross the Asian borders and then threatened public health globally in the following days. ${ }^{1,2}$ Thereafter, The World Health Organization (WHO) declared Covid-19 a Global pandemic, most of the countries closed its borders and people were quarantined. ${ }^{3}$ The novel coronavirus, named as SARS-CoV-2, spreads primarily thru human to human transmission when people are in close contact ${ }^{4}$ for this reason, businesses, schools, places of worship, restaurants closed and many social events cancelled. Isolation and inadequate information about the outbreak caused fear. Psychological distress and symptoms of mental disorders have increased during the pandemic such as Covid-19. ${ }^{5,6}$ During the 2003 SARS outbreak, post-traumatic stress disorder and depression symptoms had elevated after quarantine process in Toronto, Canada. ${ }^{7}$

The fear of getting infected affects the entire population, but it is felt more in pregnant women who are especially sensitive because of the anxiety of contamination of the fetus. Although Covid-19 pneumonia is not common in the pregnancy age group, pregnant women are vulnerable, due to physiological immunosuppression. $^{8}$

There are limited studies about psychological wellness in pregnancy during an outbreak. The exaggerated fear of encountering infection in pregnant women was shown in a study of the 2003 SARS outbreak in Hong 
Kong. ${ }^{9}$

Its known that maternal anxiety is associated with poor perinatal outcomes as preterm birth, low birth weight, small for gestational age and smaller head circumference. ${ }^{10,11}$

Although it is important to get adequate psychosocial support during pregnancy, pregnant women feel lonely because of quarentine process in a pandemic. Furthermore they also avoid visits to their physicians in fear of encountering infection in public transports or at the hospital.,

When the Covid-19 pandemic reached Istanbul-Turkey, our research team utilized the opportunity to research the psychological impact of Covid-19 on pregnant women during their visit to the our maternity ward and outpatient clinic with face to face surveys

\section{Methods:}

Participants:In our study, a total of 291 patients were included who applied for routine antenatal control or gynecologic complaints. They had no clinical symptoms of Covid-19 and had no suspicion of disease. 174 of the participants were pregnant, and 117 were non-pregnant patients. The study was designed as cross sectional study and data was obtained from both qualitative and quantitative methods between 05 May (after the start of restrictions) and 31 May (the date restrictions eased) during the Covid-19 outbreak.

Participants are exluded if they: had a history of psychiatric disorders, could not read or write in Turkish, did not agree to participate in the study, had Covid-19 symptoms and Covid-19 PCR positivity.

The study protocol was approved by the institutional ethical review board.

\section{Survey method:}

After descriptive information (age, education, working status) and obstetric history (gravida, parity number, last menstrual period) was recorded, participants were asked about the number of people living at home with them, and whether their was Covid-19 positive person amongst them, if so, if she could isolate herself from that person.

We asked the following questions in order to determine behavioral changes and concerns about pregnancy follow up during Covid-19 outbreak: 'During the COVID-19 pandemic, are you getting adequate psychosocial support?', 'Are you afraid of visiting the hospital for prenatal checks?", "If you are afraid of visiting the hospital, what is the reason?", "Are you afraid you or your baby, or both of you being infected with Covid19?", " Have you missed any pregnancy visit?", "How often do you wash your hands?", "Are you wearing a mask?", "Are you wearing gloves?"

Upon completing the above, the participants where asked to complete the Spielberger State-Trait Anxiety Inventory (STAI) form to determine the level of anxiety. The STAI is a 40-item self-report rating scale. Each statement has four scale of feelings, participants are asked to select best matching feeling, the state anxiety scales include 'not at all, somewhat, moderately so, or very much so'. Whereas the responses to the items related to trait anxiety include 'almost never, sometimes, often, and almost always'. The scale has internal consistency coefficients ranged from .86 to $.95 .^{12}$ It can vary with changes in support systems, health, and other individual characteristics. ${ }^{13}$ Validity and reliability study of the Turkish form of the scale was performed by Oner and Le Compte. ${ }^{14}$ Since the STAI is used to measure the intensity of anxiety (instead of identifying possible clinical cases), no cut-off score is recommended.

\section{Statistical analysis:}

In this study, all information obtained was entered in to statistical package for the social sciences, version 25.0, SPSS Inc, Chicago, Illinois, USA (SPSS). Descriptive statistics were used to calculate the frequency (n), percentage (\%), central tendency (mean, median\&mode) and dispersion (range, variance, SD, maximum\& minimum) for each variable when appropriate. The consistency of the data with the normal dispersion has been evaluated by the Kolmogorov-Smirnov test. Student-t test, Mann Whitney- U test, One-way ANOVA test or Chi square test were used when appropriate. 


\section{Results :}

\section{Characteristics of all participants:}

In this study, we approached 174 pregnant and 117 nonpregnant women, none of them were health care workers and diagnosed with coronavirus disease. The sociodemographic and clinical characteristics of the participants are summarized in Table 1 . Pregnant women, were younger than non pregnant women $(28,48 \pm 5,73$ vs $32,05 \pm 10,58, \mathrm{p}=0,02)$ and the number of nonemployed was higher $(24,77 \%$ vs $43,6 \%, \mathrm{p}=0,003)$. Educational status of pregnant women was lower than nonpregnant women $(\mathrm{p}=0,001)$.

The participant's who were pregnant lived with an average number of $3(\max 16)$ and non-pregnant participants lived with an an average of $4(\max 8)$ people in their house. 9 of the pregnant women said a Covid-19 patient lived with them and all of these women said they could isolate themselves from this person at home.

\section{Anxiety level and behavioral changes of all participants:}

Pregnant women had significantly higher anxiety STAI state (STAI-S) scores than the nonpregnant women $(43,67 \pm 10,77$ vs. $39,71 \pm 9,55, \mathrm{p}=0,01)$. The STAI trait (STAI-T) anxiety scores were not different between the two groups ( $43,57 \pm 8,07 \mathrm{vs} 43,07 \pm 9,41, \mathrm{p}=0,74)$. $37,9 \%$ pregnant women, $47 \%$ nonpregnant women mentioned that they could not get enough psychosocial support (Table 1).

To reduce risk of infection, $98,3 \%$ of pregnant and 98,3\% of nonpregnant women wear a mask, 54,6\% of pregnant, 59,8\% nonpregnant wear gloves, all pregnant women wash their hands more frequent than usual, just $1,7 \%$ of nonpregnant said they wash their hands as usual. Higher number of pregnant participants answered 'never' to the question; "Are you going out from your house" than nonpregnant women $(42,5 \%$ vs $27,4 \%$, $\mathrm{p}=<0,001)$ (Table1).

\section{Characteristics, anxiety level and behavioral changes of pregnants:}

$60(34,48 \%)$ of a total of 174 pregnant women were in first trimester, 53(30,45\%) were in second and $61(35,05 \%)$ were in third trimester. STAI-S and STAI-T scores were not statistically different amongst the trimester groups. Furthermore the education level of the pregnant women and whether they received psychosocial support or in which trimester they were, did not make any difference between the STAI-S scores. STAI-S scores were significantly different in the different age groups $(\mathrm{p}=0,031)$ (Table 2). Post-hoc comparisons revealed that STAI-S scores for pregnants in age groups 'over 20 years' were similar, whereas STAI-S scores of pregnants 'under 20 years' showed a significant difference when compared to pregnants in the older age groups of '20-30 year olds' $(\mathrm{p}=0,02)$ and ' $30-40$ year olds' $(\mathrm{p}=0,03)$.

Cancelling appointments of prenatal care (46,5\% of total pregnants) and the fear of going to hospital (67\% of total pregnants) is obviously high in third trimester $(46,9 \%, \mathrm{p}=<0,001 ; 41,5 \%, \mathrm{p}=0,002)$ (Table 3). Participants, who did not go to antenetal visits, stated that they were afraid of contracting Covid-19 in hospital themselves or their unborn baby or both, there was no difference between the trimester groups.

\section{Discussion}

\section{Main Findings:}

This study demonstrates that, pregnant women had significantly higher anxiety STAI-S scores than the nonpregnant women. STAI-S and STAI-T scores were not statistically different in between trimester groups. Additionally the education level of the pregnant women and whether they received psychosocial support or in which trimester they were, did not make any difference between the STAI-S and STAI-T scores. Pregnants under age 20 showed significantly different scores of STAI-S with pregnants 20-30 age group and 30-40 age group. Almost half of pregnant women stated that they never leave their home. Furthermore, fear of contracting Covid-19 caused the pregnant women to avoid going to their prenatal visits. Cancelling appointments of prenatal care and the fear of going to hospital is obviously high in third trimester, that caused poor quality of antenal care.

\section{Strengths and limitations:}


Our study is not an initial phase study, thus, it was planned that psychosocial changes of the subjects to pandemic were fully settled and could be followed up more clearly. Previous surveys were mostly conducted using online panel, but we conducted face to face survey that enable more accurate information. Our work has limitations that deserve discussion. Questionnaire limitations : M.D. Gunning et al reported that the STAI state scale reflects situation specific anxiety, but the location of the antenatal clinic in which it was completed, could change anxiety levels. ${ }^{15}$ Single-centre study : Our data may not be entirely representative of all pregnant individuals. The study was a single center study, conducted in a training and research hospital in Istanbul. Age distribution: Because of fertility age period is limited, pregnant women were younger than non pregnant women. Besides age distribution in pregnant women was homogenous.

\section{Interpretation:}

Covid-19 firstly identified and became epidemic in China and has resulted in an ongoing pandemic. ${ }^{16,17}$ The increasing numbers of death tolls, caused global fear and panic. After the first case of Covid-19, detected in Turkey, Turkish government rapidly carried out interventions and restrictions to prevent the spread of the virüs. ${ }^{18} 05$ May, the date of the beginning of our study, Turkish ministry of health announced that, total coronavirus cases in Turkey was $129,491 .{ }^{19}$ Our study was conducted after the start of restrictions in Turkey when the psychological and behavioral changes of the subjects were fully settled. A similar situation had been shown in a study, conducted in 2003 SARS outbreak in Hong Kong; with the rising in the number of cases, the level of anxiety scores also increased. The anxiety scores were highest approximately 1 month after the first SARS case was announced. Women between the 30-49 ages and less educated were more concerned. Anxiety scores of those who perceived that they were more likely to contract or die due to SARS were significantly higher. ${ }^{20}$

Perinatal anxiety is quite common and deserves clinical attention. According to a meta analyze published in 2017, which included 102 studies with total of 221,974 women, the over all prevalence for any anxiety disorder was $15.2 \%{ }^{21}$ Antenatal anxiety was associated with increased risks for preterm birth, low birth weight, earlier gestational age, and being small for gestational age, smaller head circumference. ${ }^{9}$ Also developmental delay $^{22}$, especially the development of brain structure and function disorders in children are associated with prenatal anxiety and depression. ${ }^{7,23-26}$ While we know widely about perinatal anxiety, there is a limited knowledge about psychological responses caused by a pandemic. A multi-center cross-sectional study was initiated in China to compare the mental status of pregnant women before and after the announcement of the Covid-19 epidemic. A total of 4124 pregnant women during their third trimester were examined in this cross-sectional study, using the Edinburgh Postnatal Depression Scale (EPDS). They found that, awareness of Covid-19 epidemic significantly increased the prevalence of depressive symptoms (EPDS[?]10) $(\mathrm{aRR}=1.20,95 \% \mathrm{CI}: 1.04,1.40,245 \mathrm{P}=0.01)$ and the risk of self-harm thoughts $(\mathrm{aRR}=2.85,95 \% \mathrm{CI}: 1.70$, 8.85, $\mathrm{P}=0.005)$. A linear positive association was also noted between EPDS scores and the number of new infections confirmed daily. ${ }^{27}$ Gillian A. Corbett, et al. questioned total of 71 patients in the second and third trimester of pregnancy. Half of the women who did not have anxiety before, worried about their health during the delay phase of outbreak. This anxiety was related to health of their older relatives', other children they had and then their unborn baby. ${ }^{28}$

The previous researches, compared pregnant womens' anxiety levels with before and after pandemic, not with non pregnant women, our cohort showed that pregnant women are vulnerable and they feel fear deeper. Interaction with their relatives (mother, father, friends), provides psychological support, but the necessity of social distanding did not allow this. From Calgary, Canada, 92.9\% of 1987 individuals reported feeling loneliness more than usual due to the Covid-19 pandemic. $56.6 \%$ of participants had clinically elevated anxiety and $37.0 \%$ elevated symptoms of depression. Most of the participants expressed worries about their own life and their unborn baby due to the possibilty of infection. Researchers recorded that depression and anxiety symptoms were reduced if participants could complete enough sleep time and had better social support. $^{7}$

According to a preliminary study from Turkey, mean BDI (Back Depression Index) scores, and mean BAI (The Beck Anxiety Inventory) scores were greater in the pregnant women. They interpreted that, in the 
absence of psychosocial support, adverse perinatal outcomes could occur. ${ }^{29}$ However, Mirzadeh and Khedmat also highlighted the need for psychological support for pregnant women during this pandemic ${ }^{30}$, in our study $62 \%$ of participants reported that they get adequate support, but it did not make difference on their anxiety levels.

During the course of pregnancy, maternal anxiety varies, Haddad et al. ${ }^{31}$, Teixeira et al. ${ }^{32}$ and Bhagwanani et al. ${ }^{33}$ reported that STAI-S levels were increased in the first and third trimester. Other studies have reported that STAI-S scores elevated significantly in the third trimester. ${ }^{15,34}$ The anxiety level differences between trimesters were not evaluated in an outbreak period, we found that, there was no difference of STAI-S and STAI-T levels

between trimesters.

Wearing masks, gloves, and washing hands have became daily life necessities for protection from the virus. These attitudes were experienced before, in 2003, during SARS outbreak, about $70 \%$ of women wore a mask all or most of the times, and $40 \%$ washed their hands much more frequently than before. ${ }^{35}$ Self isolation and 'not leaving home' is another reaction to Covid-19 pandemic in pregnant women. ${ }^{28}$ Furthermore, domestic transportation registrations and the intensity of Covid-19 patients in hospitals, caused the pregnant women to avoid going to their prenatal visits. It was reported former SARS outbreak, the rate of cancelling or postponement of antenatal visits were high and about half of the women decided delivering in hospitals with fewer SARS cases. ${ }^{35}$ The fear of going to hospital was obviously high in our study and half of pregnants stated that they cancelled or missed their prenatal visits. In the course of pandemic time, it is reported that, cancelling of appointments, difficulties in accessing health units, or going to physicians without a supporter caused poor quality of prenatal care. ${ }^{7}$

\section{Conclusion:}

Although anxiety can have negative effects on mental health of the entire community, especially in pregnant women it felt more and may have associated with poor perinatal outcome. Despite this, during a pandemic, obstetric services stay in the background, and obstetricians may work in other services. Being aware of the increase in anxiety and supporting pregnants by an ongoing health services is important. Governments and health systems need to be develop new policies and strategies for situations such as pandemic and catastrophes. Evaluation and disseminated use of online health services may be helpfull in patient information and empowerment.

\section{Acknowledgement:}

We wish to thank the women, researchers and clinical staff who provided significant contributions to this study

\section{Disclosure of Interest:}

The authors declare that they have no conflict of interest. Completed disclosure of interest forms are available to view online as supporting information.

\section{Contribution to authorship}

$\mathrm{BC}$ designed the study and wrote the main manuscript text. EA performed statistical analysis. FYO, BC and EA provided interpretation of the data. BC, BA, SG, HTO, AK, BT, EDPK, VM, AK interviewed with participants collected the data. All authors contributed to the final version of the manuscript.

\section{Details of Ethics:}

This study was approved by the Medical Ethical Committee of Istanbul Provincial Health Directorate Prof. Dr. Cemil Taşcıoğlu City Hospital (No:48670771-514.10;date: May 2020)

\section{Funding:}

None 


\section{References}

1. Wang C, Horby PW, Hayden FG, et al. A novel coronavirus outbreak of global 412 health concern. The Lancet 2020;395(10223): 470-473. 413

2. WHO. Clinical management of severe acute respiratory infection when Novel coronavirus (nCoV) infection is suspected: interim guidance. [Internet]. Jan 11, 2020. [https://www.who.int/publicationsdetail/clinical-management-of-severe-acute-respiratory-infection-when-novel-coronavirus-(ncov)infection-is-suspected]. Accessed Jan 20, 2020.Google Scholar

3. Chan JF-W, Yuan S, Kok K-H, et al. A familial cluster of pneumonia associated 426 with the 2019 novel coronavirus indicating person-to-person transmission: a study of 427 a family cluster. The Lancet 2020;395(10223): 514-523.

4. "Symptoms of Coronavirus"CDC - National Center for Health Statistics - Homepage. https://www.cdc.gov/coronavirus/2019-ncov/symptoms-testing/symptoms.html. last updated May 13,2020

5. Bao Y, Sun Y, Meng S, et.al. 2019-nCoV epidemic: address mental health care to empower society. Lancet. 2020;22(395):e37-e38.

6. Rajkumar RP. COVID-19 and mental health: A review of the existing literatüre. Asian J Psychiatr. 2020 Apr 10;52:102066

7. Lebel C, MacKinnon A, Bagshawe M, et.al. Elevated depression and anxiety among pregnant individuals during the COVID-19 pandemic. PsyArXiv, Apr.23 2020. Web. doi:10.31234/osf.io/gdhkt.

8. Armenti VT, Moritz MJ, Cardonick HE, et.al. Immunosuppression in Pregnancy: Choices for Infant and Maternal Health. Drugs. 2002;62(16):2361-75

9. Lee DT, Sahota D, Leung TN, Yip AS, et.al. Psychological responses of pregnant women to an infectious outbreak: A case-control study of the 2003 SARS outbreak in Hong Kong. J Psychosom Res. 2006 Nov;61(5):707-13

10. S Grigoriadis, L Graves, M Peer et.al. Maternal Anxiety During Pregnancy and the Association With Adverse Perinatal Outcomes: Systematic Review and Meta-Analysis. J Clin Psychiatry. 2018 Sep 4;79(5):17r12011

11. Schetter CD. Tanner L. Anxiety, Depression and Stress in Pregnancy: Implications for Mothers, Children, Research, and Practice. Curr Opin Psychiatry. 2012 Mar;25(2):141-8.

12. Spielberger, C. D., Gorsuch, R. L., Lushene, R., Vagg, P. R., \& Jacobs, G. A. (1983). Manual for the State-Trait Anxiety Inventory. Palo Alto, CA: Consulting Psychologists Press.

13. Elliott, TR, Shewchuk RM, Richards JS. (2001). Family caregiver problem solving abilities and adjustment during the initial year of the caregiving role. Journal of Counseling Psychology 2001;48(2):223-232

14. Oner N, LeCompte A. State-Trait Anxiety Inventory Handbook. Bogazici University Publishing, Istanbul, 1985.

15. Gunning M.D, Denison F.C, Stockley C.J, Ho S.P, Sandhu H.K, Reynolds R.M. Assessing maternal anxiety in pregnancy with the State-Trait Anxiety Inventory (STAI): issues of validity, location and participation. Journal of Reproductive and Infant Psychology. 2010; 28(3):266-273

16. Hui DS, I Azhar E, Madani TA, Ntoumi F, Kock R, Dar O, et al. The continuing 2019-nCoV epidemic threat of novel coronaviruses to global health - The latest 2019 novel coronavirus outbreak in Wuhan, China Int J Infect Dis. 2020 Feb; 91: 264-266

17. "WHO Director-General's opening remarks at the media briefing on COVID- 19". World Health Organization (WHO) (Press release). 11 March 2020. Archived from the original on 11 March 2020. Retrieved 12 March 2020.

18. Demirbilek Y, Pehlivanturk G, Ozguler ZO, Meşe EA COVID-19 Outbreak Control, Example of Ministry of Health of Turkey. Turk J Med Sci. 2020 Apr 21;50(SI-1):489-494.

19. Official Gazette of the Republic of Turkey (2006). Küresel Bir Grip Salgını (Pandemi) Konusunda Yapılması Gereken Hazırlıklar ile İlgili 2006/23 Sayılı Başbakanlık Genelgesi [online]. Website: https://www.resmigazete.gov.tr/ eskiler/2006/08/20060823.htm [accessed 17April 2020].

20. Leung GM, Ho LM, Chan KKS, Ho SY et.al. Longitudinal Assessment of Community Psychobehavioral Responses During and After the 2003 Outbreak of Severe Acute Respiratory Syndrome in Hong 
Kong Clin Infect Dis 2005 Jun 15;40(12):1713-20

21. Dennis CL, Falah-Hassani K, Shiri R Prevalence of Antenatal and Postnatal Anxiety: Systematic Review and Meta-Analysis Dennis. Br J Psychiatry. 2017 May;210(5):315-323.

22. Deave T, Heron J, Evans J, Emond A. The Impact of Maternal Depression in Pregnancy on Early Child Development. BJOG. 2008 Jul;115(8):1043-51

23. Adamson B, Letourneau N, Lebel C. Prenatal maternal anxiety and children's brain structure and function: A systematic review of neuroimaging studies. J AffectDisord. 2018 Dec 1;241:117

24. Lebel C, Walton M, Letourneau N, Giesbrecht GF et.al. Prepartum and Postpartum Maternal Depressive Symptoms Are Related to Children's Brain Structure in Preschool. BiologicalPsychiatry. 2016;80(11):859-868.

25. Sandman CA, Buss C, Head K, Davis EP. Fetal exposure to maternal depressive symptoms is associated with cortical thickness in late childhood. BiolPsychiatry. 2015;77(4):324-334.

26. Data resource center for child \& adolescent health. Guide to topics \& questions. http://www.childhealthdata.org/learn/nhis-child/topics_questions/3.3.9-2011-2012-nhisfamilyguide-to-topics-and-questions. Accessed.

27. Wu Y, Zhang C, Liu H, Duan C, Li C, Fan J. et.al. Perinatal depressive and anxiety symptoms of pregnant women along with COVID-19 outbreak in China. AJOG. Accepted Date: 6 May 2020 https://doi.org/10.1016/j.ajog.2020.05.009

28. Corbett GA, Milne SJ, Hehir MP, Lindow SW, O'connell MP Health anxiety and behavioural changes of pregnant women during the COVID-19 pandemic. Eur J Obstet Gynecol Reprod Biol. 2020 Jun; 249: $96-97$

29. Durankus F, Erson A Effects of the COVID-19 pandemic on anxiety and depressive symptoms in pregnant women: a preliminary study. J Matern Fetal Neonatal Med. Accepted 29 Apr 2020 DOI: 10.1080/14767058.2020.1763946

30. Mirzadeh M, Khedmat L. Pregnant women in the exposure to COVID-19 infection outbreak: the unseen risk factors and preventive healthcare patterns, The Journal of Maternal-Fetal \& Neonatal Medicine, (2020) DOI: 10.1080/14767058.2020.1749257

31. Haddad P.F., Morris N.F., Spielberger C.D. Anxiety in pregnancy and its relation to use of oxytocin and analgesia in labour. Journal of Obstetrics and Gynaecology. 1985; 6, 77-81.

32. Teixeira C., Figueiredo B., Conde A., Pacheco A., Costa R. Anxiety and depression during pregnancy in women and men. Journal of Affective Disorders. 2009;119, 142-148.

33. Bhagwanani S.G., Seagraves K., Dierker L.J, Lax M,. Relationship between prenatal anxiety and perinatal outcome in nulliparous women, a prospective study. J Dev Behav Pediatr. 1997; 89, 93-8.

34. Da Costa D, Larouche J, Dritsa M., Brender W. Variations in stress levels over the course of pregnancy: factors associated with elevated hassles, state anxiety and pregnancy-specific stress. Journal of Psychosomatic Research. 1999; 47, 609-621

35. Lee DTS, Sahota D, Leung TN, Yip ASK, Lee FFY, Chung TKH. Psychological responses of pregnant women to an infectious outbreak: A case-control study of the 2003 SARS outbreak in Hong Kong. J Psychosom Res. 2006 Nov; 61(5): 707-713

\section{Hosted file}

Table 1. docx available at https://authorea.com/users/339965/articles/467106-determination-ofanxiety-levels-in-pregnant-women-during-covid-19-pandemic

\section{Hosted file}

Table 2.docx available at https://authorea.com/users/339965/articles/467106-determination-ofanxiety-levels-in-pregnant-women-during-covid-19-pandemic

\section{Hosted file}

Table 3.docx available at https://authorea.com/users/339965/articles/467106-determination-ofanxiety-levels-in-pregnant-women-during-covid-19-pandemic 